\title{
Collaborative Platforms for Crowdsourcing and Consensus-Based Decisions in Multi-Participant Environments
}

\author{
Cristian CIUREA ${ }^{1}$, Florin Gheorghe FILIP $^{2}$ \\ ${ }^{1}$ Bucharest University of Economic Studies, Bucharest, Romania \\ ${ }^{2}$ Romanian Academy \& INCE, Bucharest, Romania \\ cristian.ciurea@ie.ase.ro,ffilip@acad.ro
}

The paper presents a new approach related to crowdsourcing and consensus in the multiparticipant decision-making process. Multi-participant decision-making techniques, based on consensus building models frequently assume there are not really many decision makers in the group (appropriate operations can be done by complete enumeration). The consensus building and the crowdsourcing approach in the decision-making process are described. The most wellknown top ten crowdsourcing platforms are analyzed and a comparison between them is made, in order to show existing and partially supported features.

Keywords: Consensus Building, Collaborative Decision-Making, Evaluation Criteria, Crowdsourcing Platforms.

\section{Introduction}

In [1, p. 19], the following definition of the decision was adopted:

"The decision is the result of human conscious activities aiming at choosing a course of action for attaining a certain objective (or a set of objectives). It normally implies allocating the necessary resources and it is the result of processing information and knowledge that is performed by a person (or a group of persons) who is empowered to make the choice and is accountable for the quality of the solution adopted to solve a particular problem or situation."

The individual or multi-person empowered and accountable decision taker can be supported by a human support team and/or a digital (computer-based) support system. All people involved in making and taking a decision perform their tasks in various forms of collaboration. As pointed out in [2], two or more entities collaborate, as each one working individually cannot deliver the expected output, such as a product, a service or a decision solution one can identify the following forms: a) close collaboration established among the members of the decision group, b) asymmetric or skew collaboration is a particular form of the previous one and it is established among the decision takers and their own human support team of assistants and hired consultants, and c) soft collaboration of the decision taker with commonly anonymous members of a crowd. At present, all collaboration forms are enabled and stimulated by modern ICT (Information and Communication Technology) tools, systems and platforms. In the rest of the paper we will analyze the forms of collaboration and their supporting ICT means.

The decision-making group based on consensus is a collaborative process where the participants initiate and consent to maintain a decision in the greatest interest of the entire group. The group reach this minimal feasible decision by debating several options as many as possible until all group members agree to "the final" solution, even if it is not the preferred of each member. The classic "democratic" voting might enhance conflict and suspicion, because the "losers" are perceived less powerful by the decisionmaking process. [3].

In [4] it is described a mobile crowdsourcing platform that allows users of mobile devices to post and work on decision-making processes using the most popular crowdsourcing services. The convergence of crowdsourcing and distributed mobile systems enables developing a new paradigm for universal sensing and data processing. 
2 Consensus Building and Crowdsourcing

The close collaboration is carried out by a collaborative group of humans. Such a group is characterized by the following attributes: a) congruence of methods used by members with agreed common goals, b) group effectiveness in attaining the common goals, c) group efficiency in saving member resources consumed, d) group cohesion, viewed as preserving members' willingness to collaborate in the future [5].

\subsection{Consensus Building: Basic Aspects}

The common approach in the close collaboration of the components of a collaborative group is composed of two main processes: a) consensus building, and b) a selection of a recommended solution [6]. In consensus building, the participants might need to tune their opinions by making them more similar or closer to one another in an interactive process, so that, eventually, an acceptable level of consensus is reached. In [7] it is described the consensus reaching process as composed of several activities, such as: a) individual preference representations of the participants, b) aggregating individual preferences by using various methods, c) measuring the consensus level as a distance of individual preferences either to the calculated collective one, or as the result of comparing pairs of preferences, d) implementing a correction scheme designed to increase the consensus level based either on identifying the participants whose contribution to consensus reaching is negligible or aiming to minimize the number of preference revisions.

The author of [8] presents a simple software system based on three types of algorithms, such as aggregation of precedence, clustering and simple structural analysis. The system enables the efficient conduct of expert sessions having the objective the establishment and analysis of a quasi-model related to a problem domain. The process of consensus building and selection for complex problems is commonly facilitated by multiparticipant / Group DSS (Decision Support Systems) [9].

There is an implicit basic assumption in multiperson approaches based on consensus building, namely the number of participants must be limited, so that various methods proposed would be technically applicable. Sometimes, the expertise of the components of the participants might not be sufficient and the problems could be too complex and persistent and a larger number of people could bring in the necessary knowledge and help [10].

In order to apply consensus decision making efficiently, the group decision making (GDM) should take into consideration the following recommendations [11]:

- establish how to decide inside the group;

- the facilitator has the most important role of keeping the integrity of the consensus in the group decision process;

- establish a common goal to be followed by each group participant;

- it is not a problem if someone doesn't agree, because an individual cannot stop the group to take the decision agreed by the group majority;

In Figure 1 is presented the model of using consensus in a group decision-making (GDM) process. The participants of the GDM will propose a solution from a set of alternatives and if the consensus is reached, then the proposed solution was agreed and become the final solution. But, if the consensus is not reached, then the GDM participants should search and propose another solution. 


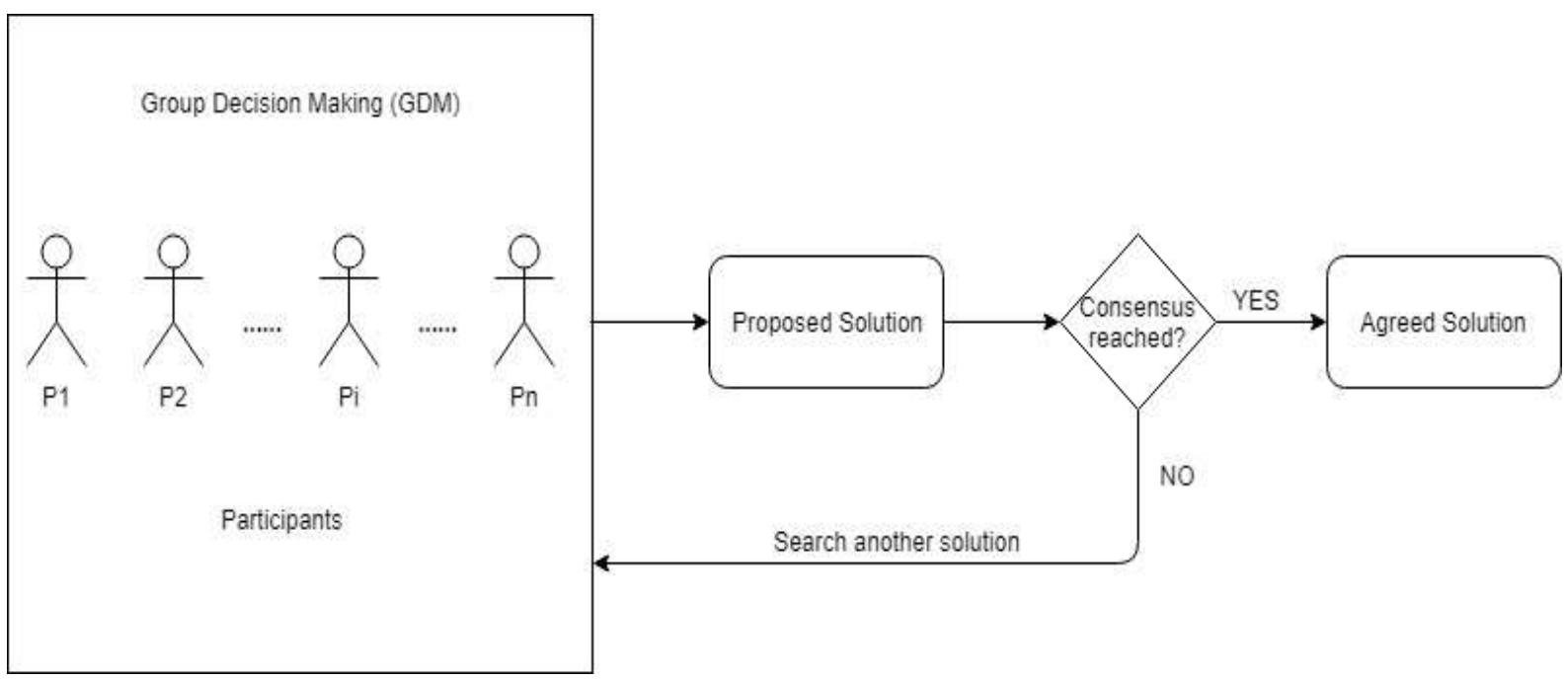

Fig. 1. The system model of consensus decision making

The collaborative decision-making process is a type of collaborative system where the decision-making process is achieved with the support of many agents that work together (communicate, coordinate, cooperate) to accomplish a common goal.
The Figure 2 below shows five reasons why the collaborative decision-making will be a hot topic in the next future.

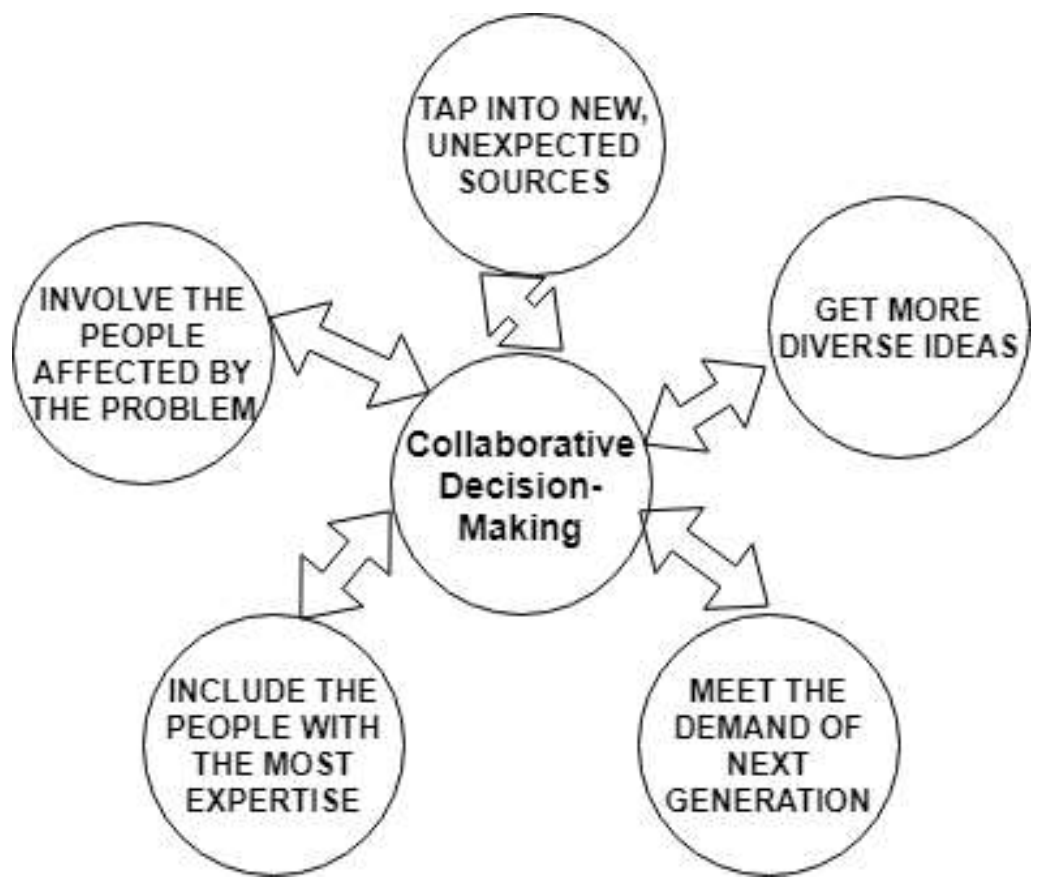

Fig. 2. Main reasons of collaborative decision-making [12]

As presented in Figure 2, in order to achieve a good collaborative decision-making process it should involve the participants directly interested in the problem to be solved, discover new sources of inspiration, extract many different ideas from the participants, adapt to the requests of the next generation and involve the most experienced persons in the process.

\subsection{Crowdsourcing in Decision-Making}

At present, crowdsourcing is ever more frequently used in various domains including collaborative decision-making [1]. In [13] it is 
articulated the term and viewed crowdsourcing as "the act of taking a job traditionally performed by a designated agent (usually an employee) and outsourcing it to an undefined, generally large group of people in the form of an open call". Therefore, the need for supporting platforms becomes obvious. Having done an extensive analysis of existing definitions and systems which were claimed to be crowdsourcing solutions, it is proposed the following integrated definition in [14]:

"Crowdsourcing is a type of participative online activity in which an individual, organization, or company with enough means proposes to a group of individuals of varying knowledge, heterogeneity, and number, via a flexible open call, the voluntary undertaking of a task. The undertaking of the task, of variable complexity and modularity, and in which the crowd should participate bringing their work, money, knowledge and/or experience, always entails mutual benefit. The user will receive the satisfaction of a given type of need, be it economic, social recognition, self-esteem, or the development of individual skills, while the crowdsourcer will obtain and utilize to their advantage that what the user has brought to the venture, whose form will depend on the type of activity undertaken."

The above authors also established a set of attributes a system should possess to qualify as a crowdsourcing one:

- "There is a clearly defined crowd;

- There exists a task with a clear goal;

- The recompense received by the crowd is clear;

- The crowdsourcer is clearly identified;

- The compensation to be received by the crowdsourcer is clearly defined;

- It is an online assigned process of participative type;

- It uses an open call of variable extent;

- It uses the Internet."

Having performed an analysis in accordance with the above criteria, the cited authors state that, out of set of systems which were commonly viewed at the moment of the study as crowdsourcing ones, several can be qualified as crowdsourcing, such as Innocentive, Threadless, AMTurk, and iStockPhoto. Also, ModCloth and Fiat Mio could be accepted even though they do not satisfy all the criteria. Other systems, such as Wikipedia or Youtube, do not qualify [14].

There are various possible uses of crowdsourcing. For example, in decisionmaking the framework is composed by the following phases [1], [15]:

- Identification of the problem to be solved or the opportunity to be exploited based on opinions and predictions collected from the crowd and task definition to facilitate intelligence phase of Simon's process model;

- Task broadcasting to the crowd, which is commonly carried-out as an open call. The crowd may be composed of either enterprise employees or customers and/or other outsiders;

- Idea generation by the crowd in the form of various action alternatives. It basically corresponds to the design phase of Simon's process model.

- Evaluation of ideas by the same members of the crowd that generated the idea or by another crowd or limited group of hired experts who may pursue the process of reaching the consensus, as described in the previous section;

- Choosing the solution through a voting mechanism.

As noticed by [16] "in crowdsourcing, a task is not performed by a designated outsourcing company or worker, but it is accomplished by the crowd". This means that an employer (sometimes called 'crowdsourcer' or 'initiator'), when using crowdsourcing, does not choose who will work on the task, but he will hand over the task to the crowd and an anonymous worker will complete it. Consequently, a platform is needed to make the soft collaboration of outsourcers (here the decision takers and, possibly, their assistants) and the contributors from the crowd.

The Figure 3 below describes the flow between the participants in the traditional 
crowdsourcing model. The "worker" receives a task from the crowdsourcing platform, which was posted by the "requestor". The "worker" and the "requestor" will communicate also outside the crowdsourcing platform, in order to clarify every detail of the task to be implemented.

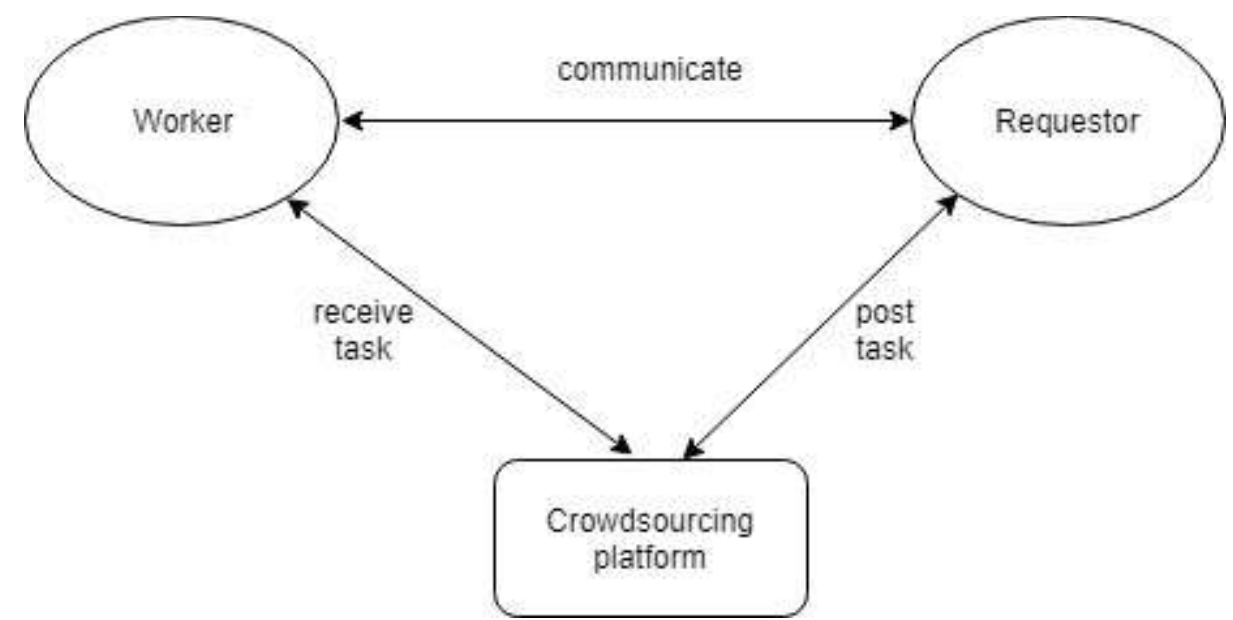

Fig. 3. The system model of classic crowdsourcing [17]

In [16] a decentralized framework for crowdsourcing is presented, where a task can be solved by a crowd of workers without relying on any third trusted institution. Crowdsourcing has gained considerable interest and adoption in the last few years, especially the platforms which utilize the human intelligence to solve complex tasks. The authors of [17] have classified the crowdsourcing systems in three main categories:

- centralized crowdsourcing systems;

- distributed crowdsourcing systems;

- blockchain-based crowdsourcing systems.

The same source describes the potential threats and malicious behaviors of crowdsourcing systems, such as the malicious requesters and workers which have different objectives to maximize their own profits. In a crowdsourcing platform should be implemented all the security features that a web application must have, in order to avoid issues, such as data leaks related to workers, requestors, projects, tasks and payments.

\section{Platforms}

\subsection{A Survey of Existing Platforms}

According to [18], in the current section we describe a list of top ten crowdsourcing platforms that can assist the user in designing and creating a product, from developing an idea to creating a 3D object or a full real product.

Some of these platforms were built to help the decision-making process in various contexts, from growing an idea to designing and creating a product or for every intermediate stage.

The following platforms are covering all of these situations [18]:

- Idea Bounty

- (https://www.crunchbase.com/organizati on/idea-bounty): it is a crowdsourcing platform for creative ideas and marketing solutions that assist to carry your idea from the seed stage to the end;

\section{- OpenIdeo}

- (https://www.openideo.com/) is a platform that can speed up the impact by gathering the right people together, being mainly oriented towards philanthropic projects, growing ideas that can make the world better;

\section{- Innocentive}

(https://www.innocentive.com/) offers a validated and successful approach to find various ideas and solutions, being another "problem solving" platform that connect solution seekers; 


\section{- CrowdSpring}

(https://www.crowdspring.com/) is a great tool that allow you to write down the idea and wait for submissions from the freelancers on the platform; after that, you can choose the favorite and you'll only pay for that one:

- 99Designs (https://99designs.co.uk/) is more an artistic platform, mostly oriented on graphic design than product design, where more than 1 million professionals are working to implement the most attractive solution;

- Cad Crowd

- (https://www.cadcrowd.com/) is a platform where you can freelance 3D CAD (Computer Aided Design), 3D modeling, but the winner can be hired in a more traditional way, for example paying for hours spent on the project;

- DesignCrowd

(https://www.designcrowd.com/) is a platform for the best freelance design, where you can find designers based on different filters, such as specialty, country, earnings, percentage of jobs awarded etc.;

\section{- MicroWorkers}

(https://www.microworkers.com/) is a crowdsourcing platform for micro-tasks, where the user can choose from any of the ready-to-use templates available on the platform;

- Mechanical Turk / mTurk

- (https://www.mturk.com/) is a crowdsourcing platform created by Amazon to outsource different processes and jobs offered by companies to a geographical distributed workforce that can realize these tasks in a virtual manner;
- (https://www.upwork.com/) is a platform that helps to find various profiles, such as web developers, software designers, proficient translators, and so on, and the platform enables the possibility to keep in touch with these professionals, so that one can develop a strong relationship except the freelancing;

\subsection{Comparing Platforms}

Based on the stages of the crowdsourcing process described in [19], we have considered the following criteria to classify the crowdsourcing platforms presented above:

- registration and specification;

- initialize crowdsourcing request;

- carry out crowdsourcing request;

- complete crowdsourcing request;

The registration and specification criteria means the feature of the platform that allow the registration of new users and the specification of the problem to be solved.

The criteria related to initialization of the crowdsourcing request means the capability to create one or more requests on the platform in order to ask users to solve a problem.

Carrying out the crowdsourcing request refers to the possibility to establish a good communication, directly on the platform, between workers and requestors, in order to send and receive tasks and solutions.

The criteria meeting the crowdsourcing request refer to the feature of the platform that allows the workers to deliver the solutions to the requestors who have implemented the requested problem.

Table 1 below presents a comparison of the top ten crowdsourcing platforms available on the market based on the four criteria defined earlier.

\section{- oDesk.com / Elance}


Table 1. Comparison between crowdsourcing platforms

\begin{tabular}{|l|l|c|c|c|c|}
\hline No. & $\begin{array}{l}\text { Platform } \\
\text { name }\end{array}$ & $\begin{array}{l}\text { Registration } \\
\text { and } \\
\text { specification }\end{array}$ & $\begin{array}{l}\text { Initialize } \\
\text { crowdsourcing } \\
\text { request }\end{array}$ & $\begin{array}{l}\text { Carry out } \\
\text { crowdsourcing } \\
\text { request }\end{array}$ & $\begin{array}{l}\text { Complete } \\
\text { crowdsourcing } \\
\text { request }\end{array}$ \\
\hline 1 & Idea Bounty & + & + & + & + \\
\hline 2 & OpenIdeo & + & $+/-$ & $+/-$ & $+/-$ \\
\hline 3 & Innocentive & + & $+/-$ & +- & $+/-$ \\
\hline 4 & CrowdSpring & + & + & $+/-$ & $+/-$ \\
\hline 5 & 99Designs & + & $+/-$ & $+/-$ & $+/-$ \\
\hline 6 & Cad Crowd & + & $+/-$ & $+/-$ & $+/-$ \\
\hline 7 & DesignCrowd & + & $+/-$ & +- & $+/-$ \\
\hline 8 & MicroWorkers & + & $+/-$ & $+/-$ & $+/-$ \\
\hline 9 & $\begin{array}{l}\text { Mechanical } \\
\text { Turk }\end{array}$ & + & + & $+/-$ & $+/-$ \\
\hline 10 & $\begin{array}{l}\text { oDesk.com / } \\
\text { Elance }\end{array}$ & + & $+/-$ & $+/-$ & \\
\hline
\end{tabular}

Legend:

- + feature exists,

- +/- partially supported,

- - feature does not exist,

- $\mathrm{NA}=$ not applicable to use case

As seen from Table 1, all of the crowdsourcing platforms presented satisfy the criteria defined.

In [20] an analysis of paid and unpaid crowdsourcing platforms is made, depending on the incentive given to the crowd workers.

The examples of paid platforms are the Amazon Mechanical Turk (mTurk), CrowdFlower, the Microworkers platform and Bazaar.

The unpaid platforms examples are Crowd $4 U$, Bossa, PyBossa, Zooniverse and CrowdButton.

\subsection{Selecting a Platform}

In selecting the service of a platform, the general set of criteria presented in [21] can be used;

- Adequacy: informational transparency, accuracy of expected results, robustness to errors and low quality uncertain input data, response time;

- Quality of implementation: scalability, flexibility, functional transparency, documentation completeness;

- Delivery quality: price, service delivery time, provider's general reputation, easy adaptation, degree of dependence on the technical assistance from the provider's specialists for implementation and usage. In such situations when more than one person are involved in choosing the service of the platform, the simple procedure for consensus building inspired by [22] and presented in section 2.3.4.2 of [1] is recommended. It is based on the IPV (Intensity-Polarity-Voting) model. In case there is a moderator in the selection group, the procedure presented in [23] could be used.

\section{Conclusions}

The implicit common assumption concerning the limited number of persons in multiparticipant decision-making approaches based on consensus-building systematic procedures, so that various methods proposed would be technically applicable, was stated above in section 2.1. Moreover, the identification or design of the set of alternatives is a decision problem per se. If such a problem is not solved in a satisfactory way, there is the risk of applying systematic consensus building and selection procedures to an incomplete set and of eventually choosing the best one of several poor candidate alternatives. In such a situation 
crowdsourcing may help and the platforms enable the interaction of the crowdsourcer unit and the people of the crowd.

The group decision-making (GDM) is characterized by the fact that participants / group members should agree on the same decision. This approach can be realized based on the voting mechanism, where each group member can choose between a collections of alternatives.

When discussing about voting, we should take into consideration the impact on modern information and communication technologies (ICTs) on the voting process, such as the implementation of an electronic voting mechanism. Such a system can be implemented at large scale (national elections) only after a full audit and security analysis.

Besides voting, other methods are also presented, such as judgment aggregation, resource allocation, group argumentation, in the third chapter of [1].

\section{Acknowledgement}

This paper is an extended version of the article presented at the 18th International Conference on Informatics in Economy, IE 2019, 30-31 May 2019, Bucharest, Romania. The research will continue in the frame of the Joint Research Project "Multiparticipant Cooperative Decision-Making: Consensus Building vs. Crowdsourcing-based Decisions" between the Romanian Academy and the Polish Academy of Sciences.

\section{References}

[1] F. G. Filip, C. B. Zamfirescu, C. Ciurea, Computer Supported Collaborative Decision-Making. Springer, 2017.

[2] S. Y. Nof, J. Ceroni, W. Jeong, M. Moghaddam, Revolutionizing Collaboration through e-Work, e-Business and e-Service. Springer-Verlag, Berlin, 2015.

[3] Consensus Decisioning - How to Find Minimal Viable Decisions, Available at: http://www.plays-inbusiness.com/consensus-decisioning-how- to-find-minimal-viable-decisions/, Accessed: 06.04.2019

[4] T. Yan, M. Marzilli, R. Holmes, D. Ganesan, M. Corner, "Demo Abstract: mCrowd - A Platform for Mobile Crowdsourcing," Proceedings of the 7th ACM Conference on Embedded Networked Sensor Systems, November 4-6, 2009, pp. 347-348.

[5] R. O. Briggs, G. L. Kolfschoten, de G.-J. Vrede, "A six-layer model of collaboration." In: Nunamaker J.F., Romano Jr N. C., Briggs R. O. (eds). Collaborative Systems: Concept, Value, and Use. Routledge, Taylor \& Francis Group, London, 2015, pp. 211-227.

[6] G. Kou, X. Chao, Y. Peng et al, "Intelligent collaborative support system for AHPgroup decision making," Studies in Informatics and Control, vol. 26, no. 2, 2017, pp. 131-142.

[7] Y. Dong, Q. Zha, H. Zhang, G. Kou et al, "Consensus reaching in social network group decision-making: research paradigms and challenges," KnowledgeBased Systems, vol. 162, 2018, pp. 3-13.

[8] J. W. Owsiński, "A Simple Software System for Eliciting Structured Sets of Notions from a Group of Experts (Methods and Experiences)." In: Schader M., Gaul W. (eds) Knowledge, Data and ComputerAssisted Decisions. NATO ASI Series (Series F: Computer and Systems Sciences), vol. 61. Springer, Berlin, Heidelberg, 1990, pp. 369-378.

[9] F. G. Filip, "Decision Support and Control for Large-scale Complex Systems," Annual Reviews in Control, vol. 32, no. 1, 2008, pp. 62-70.

[10] F. G. Filip, "DSS - A Class of Evolving Information Systems", In G. Dzemyda et al (eds.) "Data Science: New Issues and Applications", Springer, 2020 (in press).

[11] S. Nichols, 5 Tips for Using Consensus Decision-Making Effectively, 2015, Available at: https://mncampuscompact. org/resource-posts/5-tips-for-usingconsensus-decision-making-effectively/, Accessed: 01.07.2019. 
[12] 5 Reasons Why Collaborative DecisionMaking is the Future, 2019, Available at: https://www.discuto.io/en/blog-entry/5reasons-why-collaborative-decisionmaking-future, Accessed: 01.07.2019.

[13] J. Howe, "The rise of crowdsourcing", Wired magazine, vol. 14, no. 6, 2006, pp. 1-4.

[14] E. Estelles Arolas, F. González-LadrónDe-Guevara, "Towards an integrated crowdsourcing definition, Journal of Information Science, vol. 32, no. 2, 2012, pp. 189-200. doi: 10.1177/0165551512437638.

[15] C. M. Chiu, T. P. Liang, E. Turban,'What can crowdsourcing do for decision support?" Decision Support Systems, vol. 65, 2014, pp. 40-49.

[16] M. Hirth, T. Hoßfeld, Tran-Gia, "Anatomy of a crowdsourcing platform Using the example of Microworkers.com," 2011 Fifth International Conference on Innovative Mobile and Internet Services in Ubiquitous Computing, 2011, pp. 1-7, doi: 10.1109/imis.2011.89

[17] M. Li et al., "CrowdBC: A BlockchainBased Decentralized Framework for Crowdsourcing," in IEEE Transactions on Parallel and Distributed Systems, vol. 30, no. 6, June 2019, pp. 1251-1266.

[18] H. Bensoussan, 13 Best crowdsourcing platforms for product design, Available at: https://www.sculpteo.com/blog/2016/10/1 9/13-best-crowdsourcing-platforms-forproduct-design/, Accessed: 06.04.2019

[19] M. Vukovic, "Crowdsourcing for Enterprises," Proceedings of the 2009 Congress on Services - I, IEEE Computer Society, July 06 - 10, 2009, pp. 686-692.

[20] R. M. Borromeo, M. Toyama, "An investigation of unpaid crowdsourcing", Human-centric Computing and Information Sciences, Springer, Vol. 6, No. 11, 2016.

[21] F. G. Filip, "A decision-making perspective for designing and building information systems," International Journal Computers Communications \& Control, vol. 7, no. 2 (June), 2012, pp. 264272.

[22] A. Coman, "Competence, power and conflict in group decision making" Human Systems Management, vol. 15, no. 4, 1996, pp. 245-255.

[23] J. Kacprzyk, S. Zadrożny. "Reaching consensus in a group of agents: supporting a moderator run process via linguistic summaries". In: M. Collan, J. Kacprzyk J. (eds) Soft Computing Applications for Group Decision-Making and Consensus Modeling. Studies in Fuzziness and Soft Computing, vol. 357, 2018, Springer, Cham.

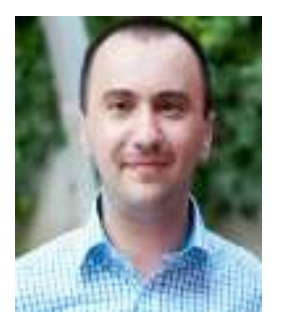

Cristian CIUREA is Associate Professor at the Department of Economic Informatics and Cybernetics at Bucharest University of Economic Studies. He has graduated the Faculty of Economic Cybernetics, Statistics and Informatics at the Bucharest University of Economic Studies in 2007. He has a master in Informatics Project Management (2010) and a PhD in Economic Informatics (2011) at the University of Economic Studies, Bucharest. Cristian has a solid background in computer science and is interested in collaborative systems related issues. Other fields of interest include software metrics, data structures, object oriented programming, windows applications programming, mobile devices programming and testing process automation for software quality assurance. 


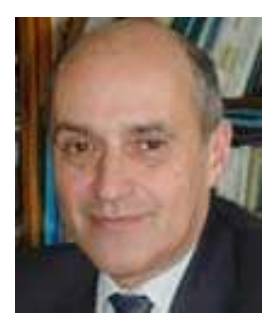

Florin Gheorghe FILIP took his MSc and PhD in control engineering at the TU "Politehnica" of Bucharest. In 1991 he was elected member of the Romanian Academy (RA). He was a scientific researcher (1970-1990) and managing director (1991-1997) of the National R\&D Institute in Informatics (ICI) in Bucharest and a part-time researcher at the National Institute of Economic Researches (INCE) of the RA (2004-2019), also the director of the Library of the Academy (2009-2017). He was elected vice-president of RA in 2000 and re-elected in 2002 and 2006. He is the chair of "Information Science and Technology" section of the Academy (elected in 2010, reelected in 2015). His main scientific interests include large-scale systems, decision support systems, technology management and foresight. 